\title{
Algebraic Topology-Based Image Deformation: A Unified Model
}

\author{
Layachi Bentabet ${ }^{*}$ and Djemel Ziou ${ }^{+}$ \\ * Computer Science Department, Bishop's University, Lennoxville, Canada \\ + Départment d'informatique, Université de Sherbrooke, Sherbrooke, Canada
}

Received 20 December 2004; accepted 6 June 2005

\begin{abstract}
In this paper, a new method for image deformation is presented. It is based upon decomposition of the deformation problem into basic physical laws. Unlike other methods that solve a differential or an energetic formulation of the physical laws involved, we encode the basic laws using computational algebraic topology. Conservative laws are translated into exact global values and constitutive laws are judiciously approximated. In order to illustrate the effectiveness of our model, we deal with both small- and large-scale deformation, utilizing elasticity theory and the viscous fluid model, respectively. The proposed approach is validated through a series of tests on optical flow estimation and image registration.
\end{abstract}

Key Words: image deformation, EDP, basic physical laws, computational algebraic topology, viscous fluid model, elasticity, optical flow, image registration.

\section{Introduction}

Physics-based deformation has been gaining in popularity since the introduction of active contours by Kass et al. [1]. The physics-based deformation problem is solved by combining the basic physical laws that constitute the fundamental equations of continuum mechanics. These equations may be developed in two separate but essentially equivalent formulations. One, the integral or global form, derives from a consideration of the basic principles being applied to a finite volume of the material. The tessellation of space into finite volumes using the finite elements method (FEM) or the finite volumes method (FVM) gives rise to discrete equations that can be directly implemented if the physical space is discrete in nature, as in the case of an image. The other, differential or field approach, involves equations resulting from the basic principles being applied to a very small (infinitesimal) element of volume. As a result of the continuum assumption, field quantities such as velocity and displacement which reflect the mechanical or cinematic properties of continuum bodies are expressed mathematically as continuous functions, or at worst as piecewise continuous functions, of the space and time variables. Moreover, the derivatives of such functions, if they are considered at all, should be continuous. In practice, it is often proposed to derive the field equations from their global counterparts instead of using the global forms directly. The convenience of the differential forms often argues in favour of this. However, the major drawback of such an approach when dealing with the deformation problem is that the description of the material will be accurate only if the

Correspondence to: <lbentabe@ubishops.ca>

Recommended for acceptance by $<$ Perales F., Draper B.>

ELCVIA ISSN: 1577-5097

Published by Computer Vision Center / Universitat Autonoma de Barcelona, Barcelona, Spain 
displacement and velocity fields vary slowly over the size of the elements used [2]. This drawback arises directly from the use of a differential formulation of the physics-based laws involved. Indeed, field equations in a differential formulation are subject to restrictions imposed by derivability, restrictions that are not related to the physical phenomenon investigated.

In this paper we introduce a new formulation of physics-based image deformation. The proposed formulation is based on the computational algebraic topology (CAT) based image model introduced by Ziou and Allili [3]. The CAT-based image model offers efficient tools that allow the encoding of global values arising from physical laws over volumes, surfaces, etc. In our model, we propose to derive the equations governing the deformation directly for a discrete volume, not for an infinitesimal point, using the basic laws instead of the differential equations. In fact, we will show that there is no need to write the equilibrium balance for an infinitesimal point, thereby introducing a differentiability restriction, when an equilibrium holds for a whole region. In this sense, we use a real discrete formulation where the equilibrium is expressed in finite terms. Consequently, the CAT-based image deformation can be applied whenever a variable is not differentiable, for example when the displacement field undergoes large variations. The proposed model is profoundly different from the method presented in the literature, since it requires neither energetic functional nor their differentiations to describe image deformation.

Both the small- and large-scale deformation cases will be taken into account. Small-scale deformation is carried out by assuming the image to be a perfect elastic solid; large-scale deformation is achieved by assuming the image to be a perfect viscous fluid. The proposed approach is validated through a series of tests on optical flow estimation and neuroanatomy registration. The paper is organized as follows. In the next section we summarize the CAT-based image model. Section 3 is devoted to some physical concepts that are useful in understanding the conservation of momentum principle and decomposing the deformation problem into basic laws. In section 4, the CAT-based image model is used to encode the physical laws. Applications and experimental results are presented in section 5. Finally; we provide a conclusion in section 6.

\section{The CAT-based image model}

In this section we give a brief overview of the CAT-based image model introduced by Ziou and Allili [3]. In this model, an image is composed of two distinctive parts: the image support and some field quantity associated with it. The image support is a complex of unit cells, usually called pixels. A pixel of dimension $q$ is called a $q$-pixel. Hence, the pixel $\gamma \subset \mathfrak{R}^{2}$ in Figure 1 is a 2-pixel. The boundaries of $\gamma$ are 1-pixels, referred to by us as the 1 -faces of $\gamma$. Similarly, the boundaries of each 1-pixel are 0 -pixels, which we refer to as 0 -faces.

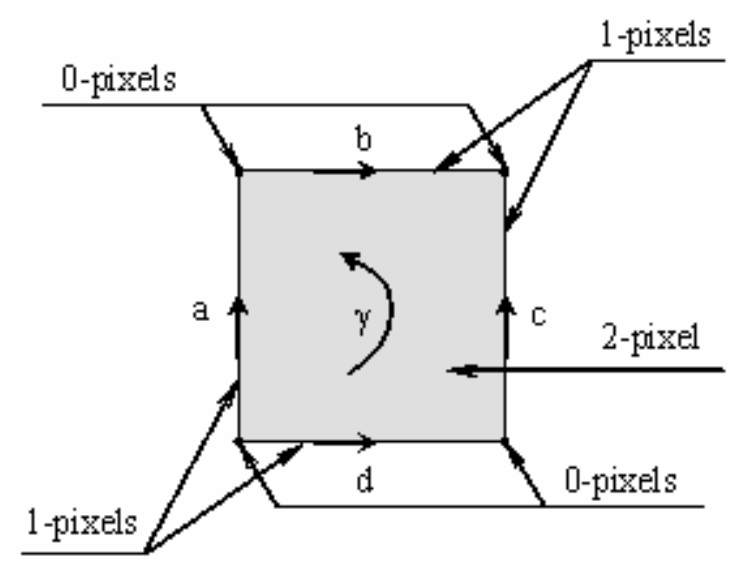

Fig. 1: A 2-pixel $\gamma$ and its boundaries

A natural orientation is assumed in order to perform algebraic operations on the fields associated with each pixel (see Figure 1). Suppose that $\gamma$ denotes a particular positively oriented $q$-pixel. It is natural to 
denote the same pixel with opposite orientation by $-\gamma$. A cubical complex in $\Re^{n}$ is a finite collection $\boldsymbol{K}$ of $q$ pixels such that every face of any pixel in $\boldsymbol{K}$ is also a pixel in $\boldsymbol{K}$. In addition, the intersection of any two pixels in $\boldsymbol{K}$ is either empty or a face.

In order to write the image support in algebraic form, we introduce the concept of chains. Any set of oriented $q$-pixels of a cubical complex can be written in algebraic form by attributing to them the coefficient 0,1 or -1 , indicating respectively that they are not in the set or should or should not be taken with positive orientation. Given a topological space $X \subset \mathfrak{R}^{n}$ in terms of a cubical complex, we get a free Abelian group $C_{q}(X)$ generated by all the $q$-pixels. The elements of this group are called $q$-chains and they are formal linear combinations of $q$-pixels. A formal expression for a $q$-chain $c_{q}$ is $c_{q}=\sum_{\gamma_{i} \in K} \lambda_{i} \gamma_{i}$, where $\lambda_{i} \in Z$.

The last step needed for the description of the image support is the introduction of the concept of a boundary of a chain. Given a $q$-pixel $\gamma$, we define its boundary, $\partial \gamma$, as the ( $q$-1)-chain corresponding to the alternating sum of its $(q-1)$-faces. The sum is taken according to the orientation of the (q-1)-faces relative to the orientation of the $q$-pixel. We say that a ( $q$-1)-face of $\gamma$ is positively oriented relative to the orientation of $\gamma$ if its orientation is compatible with the orientation of $\gamma$. By linearity, the extension of the definition of a boundary to arbitrary $q$-chains is easy. For instance, in Figure 1, the boundary of the 2-pixel $\gamma$ is $-a-b+c+d$.

In order to model the pixel quantity over the image plane, we look for an application $f_{q}: C_{q}(X) \rightarrow \mathfrak{R}^{n}$, which associates a global quantity with all $q$-pixels, where $q \leq n$. The application must satisfy the following condition:

$$
f_{q}\left(\sum_{i} \lambda_{i} \gamma_{i}\right)=\sum_{i} \lambda_{i} f_{q}\left(\gamma_{i}\right)
$$

which means that the sum of the quantities generated within each $q$-pixel is equal to the quantities generated within all $q$-pixels. The resulting application $f_{q}$ is called a $q$-cochain and may be any mathematical entity, such as a scalar or a vector that is defined over our complex.

Finally, let's define a generic operation that can be instantiated depending on the problem we are dealing with. The generic operation should specify the algebraic relationship between the quantities (i.e., cochains) associated with the faces of a $q$-pixel. It should be recalled that the boundary operator gives the relationship between the $(q-1)$-chain and the q-chain. Similarly, the relationship between the $q$-cochain and the $(q+1)$ cochain is given by the coboundary operator $\delta_{q}: C^{q} \rightarrow C^{q+1}$, where $C^{q}$ is the Abelian group of $q$-cochains. Given a $(q+1)$-chain $\gamma$, this operator is defined by

$$
\delta_{q} f_{q}(\gamma)=f_{q}\left(\partial_{q+1} \gamma\right)
$$

The coboundary is defined as the signed sum of the physical quantities associated with the $q$-faces of $\gamma$. The sum is taken according to the relative orientation of the $q$-faces of the $(q+1)$-pixels of $\gamma$.

\section{Physical Modeling of the Deformation Problem}

The first step consists of deriving the conservation of momentum law, which establishes the relationship between the external forces applied to a material and the resulting deformation. Consider a material, $M$, subject to a system of external forces, denoted by $\vec{F}^{\text {ext }}$. A system of internal forces, $\vec{F}^{i n t}=\iiint_{\Omega} \vec{\nabla} \cdot \sigma d V$, will be developed to counterbalance $\vec{F}^{\text {ext }}$. In order to describe the dynamic behaviour of the material, we use Newton's second law, which states that the resultant force acting on a body, with a density of material $\rho$, moving at velocity $\vec{v}$ is equal to the rate of change over time of the linear momentum $\rho \vec{v}$. Hence 


$$
\frac{d}{d t} \iiint_{\Omega} \rho \vec{v} d V=\vec{F}^{i n t}+\vec{F}^{e x t}=\iiint_{\Omega} \vec{\nabla} \cdot \sigma d V+\iiint_{\Omega} \rho \vec{b} d V
$$

where $\vec{b}$ describes the acceleration field and $\sigma=\left\lfloor\sigma_{i j}\right\rfloor$ is the 3 by 3 stress tensor. The elements $\left\lfloor\sigma_{i j}\right\rfloor$ of the stress tensor are defined as the limit of the internal force per unit of area as the area shrinks to zero [2] (see figure 2).

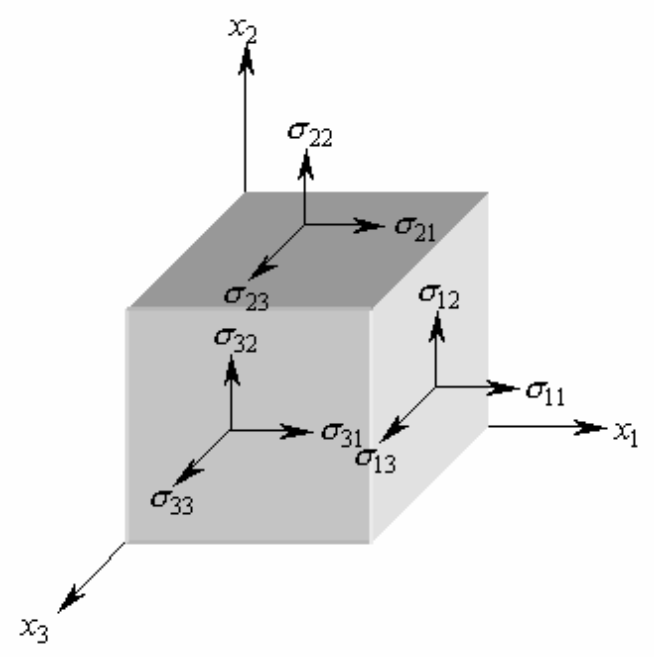

Fig. 2: Stress components

In what follows, we will focus on the equilibrium state of the material. Hence, solving the deformation problem will be equivalent to determining the material state after the deformation has been achieved. For an elastic material, equilibrium is reached when $\vec{v}=\overrightarrow{0}$. A viscous fluid is said to be in equilibrium if $\frac{d \vec{v}}{d t}=\overrightarrow{0}$. Hence, the conservation of momentum equation for both an elastic material and a viscous fluid can be rewritten at equilibrium as follows:

$$
\overrightarrow{\mathrm{F}}^{\text {int }}+\overrightarrow{\mathrm{F}}^{\mathrm{ext}}=\iiint_{\Omega}(\vec{\nabla} \cdot \sigma+\rho \vec{b}) d V=\overrightarrow{0}
$$

Note that this equation is a conservative equation, which provides a direct link between the external forces $\iiint_{\Omega} \rho \vec{b} d V$ and the internal forces $\iiint_{\Omega} \vec{\nabla} \cdot \sigma d V$.

The stress tensor is related to the strain tensor by the constitutive law which is local by nature and which defines the behaviour of the material when subject to stress. We propose to use a unified formulation of Hookes law that is valid for both elastic materials and viscous fluids. This formulation is given as follows:

$$
\sigma=[\lambda(\operatorname{tr}(\varepsilon))-\kappa p] I d+2 \mu \varepsilon
$$

where $\lambda, \mu$ and $\kappa$ are material constants, $p$ is the pressure, $t r$ is the trace operator, Id is the identity tensor, and $\varepsilon$ is the strain tensor. The Strain defines the change in configuration variable in a given direction and is given [4]: 


$$
\varepsilon_{i j}=\frac{1}{2}\left[\frac{\partial \psi_{i}}{\partial x_{j}}+\frac{\partial \psi_{j}}{\partial x_{i}}\right], \quad i, j=1,2,3
$$

where the configuration variable $\vec{\psi}$ is instantiated depending on the problem we are dealing with. It describes the displacement in case of elastic materials and the velocity in case of viscous fluids. In the reminder of this paper, we will consider another tensor $d$, where

$$
d=\vec{\nabla} \vec{\psi}=\left(\left(\vec{\nabla} \psi_{1}\right)^{T},\left(\vec{\nabla} \psi_{2}\right)^{T},\left(\vec{\nabla} \psi_{3}\right)^{T}\right)
$$

It is easy to see that $\varepsilon$ is the symmetric part of $d$, that is, $\varepsilon=\frac{d+d^{T}}{2}$.

Equations (2), (3), and (5) express the three steps needed to describe how the external forces are related to the configuration variable. As shown in figure 3, equation (2) links the external forces to the internal forces, expressed in terms of stress; equation (3) links the stress to the strain; and equation (5) links the strain and the configuration variable.

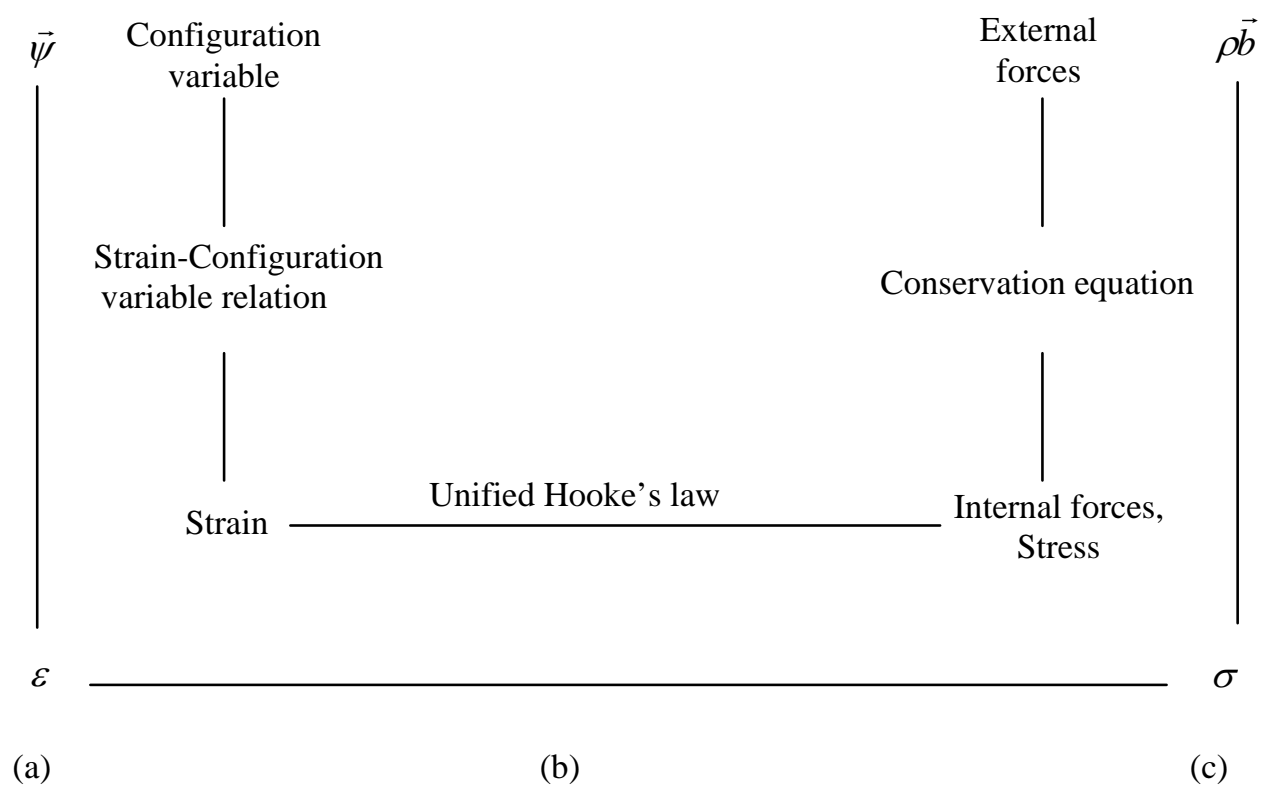

Fig. 3: a) kinematic equation, b) constitutive equation, c) conservative equation

\section{CAT-based Formulation of the Deformation Problem}

The conservative laws involved are: the conservation of momentum in equation (2) linking the external forces to stress; and the strain-configuration variable relation in equation (5). In the context of CAT, the configuration variable and the strain quantities are associated with a complex describing the image support configuration. This complex is called $K^{p}$ and it is positioned in such a way that its 0-pixels are centered on the image pixels (figure 4-a). Besides, the external forces and stress which describe the dynamic state of the material are associated with a dual complex $K^{s}$. This complex is positioned in such a way that its 2-pixels coincide with the image pixels (figure 4-a). A 2-pixel $\gamma_{F}$ from $K^{s}$ intersects four 2-pixels of $K^{p}$. This construction allows us to write the equilibrium equation for each 2-pixel $\gamma_{F}$. This involves examining and 
collecting the contributions of each portion of 2-pixels from $K^{p}$ surrounding $\gamma_{F}$. In this way, equilibrium relations are established over $\gamma_{F}$ directly in a discrete form, without approximation.

In order to encode the strain-configuration variable relation in equation (5), consider a 2-pixel $\gamma_{P}$ of $K^{p}$ as shown in figure 4-b. The strain tensor $d$ can be modeled as a 1-cochain $\mathbf{D}_{1}$ positioned on the 1-faces $\gamma_{D i}$ of $\gamma_{P}$ with $\partial \gamma_{D i}=x_{*}-x_{\#}$ (figure 4-c). Thus

$$
\mathbf{D}_{1}^{i}\left(\gamma_{D_{i}}\right)=\int_{\gamma_{D i}} d d=\int_{X_{\#}}^{X_{*}} \vec{\nabla} \vec{\psi} d l
$$

Since the configuration variable is known only at the 0 -faces of $\gamma_{P}$, it is modeled as a 0 -cohain, noted $\boldsymbol{\Psi}_{0}$, positioned on the 0 -faces $\partial \gamma_{D_{i}}$. By evaluating the line integral in equation (6), it could be shown that the cochain $\mathbf{D}_{1}$ is the coboundary of $\boldsymbol{\Psi}_{0}$. So that

$$
\mathbf{D}_{1}^{i}\left(\gamma_{D}\right)=\delta \boldsymbol{\Psi}_{0}^{i}\left(\gamma_{D_{i}}\right)=\boldsymbol{\Psi}_{0}^{i}\left(\partial \gamma_{D_{i}}\right)=\boldsymbol{\Psi}_{0}^{i}\left(x_{*}\right)-\boldsymbol{\Psi}_{0}^{i}\left(x_{\#}\right)
$$

which is a discrete representation of equation (5).

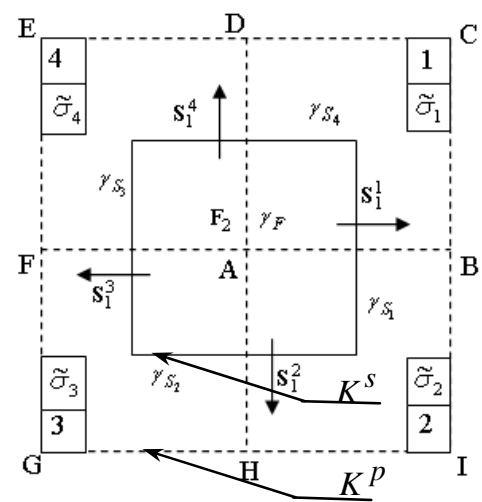

(a)

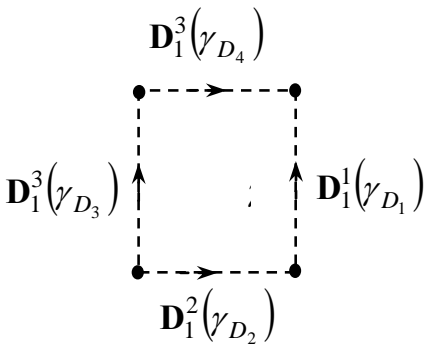

(b)

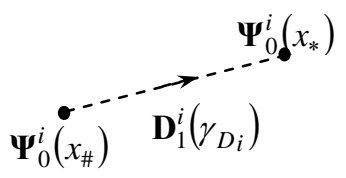

(c)

Fig. 4: a) Complexes $K^{p}$ and $K^{s}$, b) cochain $\mathbf{D}_{1}$, c) cochain $\boldsymbol{\Psi}_{0}$

In order to express the conservation of momentum in equation (2), consider a 2-pixel $\gamma_{F}$ from $K^{S}$ as shown in figure 4-a. The external forces over the surface of $\gamma_{F}$ are expressed as a 2-cochain $\mathbf{F}_{\mathbf{2}}\left(\gamma_{F}\right)$. In the equilibrium state, this cochain is calculated from equation (2) by:

$$
\mathbf{F}_{2}\left(\gamma_{F}\right)=\iint_{\gamma_{\mathrm{F}}} \rho \vec{b} d S=-\iint_{\gamma_{\mathrm{F}}} \vec{\nabla} \cdot \sigma d S
$$

Applying the divergence theorem on equation (8), we have

$$
\mathbf{F}_{2}\left(\gamma_{F}\right)=-\int_{\partial \gamma_{F}} \sigma \vec{n} d l=-\sum_{i=1}^{4} \int_{\gamma_{S_{i}}} \sigma \vec{n}_{S_{i}} d l
$$

where $\gamma_{S_{i}}$ is the $i^{\text {th }} 1$-face of $\gamma_{F}$ and $\vec{n}_{S_{i}}$ is the normal vector to $\gamma_{S_{i}}$. This allows us to define the stress tensor in equation (9) as a 1-cochain, as follows: 


$$
\mathbf{S}_{1}^{i}\left(\gamma_{S_{i}}\right)=\int_{\gamma_{S_{i}}} \sigma \vec{n}_{S_{i}} d l
$$

The 1-cochain $\mathbf{S}_{1}^{i}$ is positioned on the 1-face $\gamma_{S_{i}}$. The cochain $\mathbf{F}_{2}$ is the coboundary of $\mathbf{S}_{1}$, so that

$$
\mathbf{F}_{2}\left(\gamma_{F}\right)=-\delta \mathbf{S}_{1}\left(\gamma_{F}\right)=-\mathbf{S}_{1}\left(\partial \gamma_{F}\right)=-\sum_{i=1}^{4} \mathbf{S}_{1}^{i}\left(\gamma_{S_{i}}\right)
$$

which is the discrete representation of equation (2).

Since $\gamma_{F}$ intersects four 2-pixels of $K^{P}$, we use four approximation functions $\tilde{\sigma}^{1}, \tilde{\sigma}^{2}, \tilde{\sigma}^{3}$ and $\tilde{\sigma}^{4}$, which describe the stress tensors on each 2-pixel surrounding $\gamma_{F}$. These functions are used to build the stress cochains in equation (11):

$$
\begin{aligned}
& \mathbf{S}_{1}^{1}\left(\gamma_{S_{i}}\right)=\int_{0}^{\frac{\overline{A D}}{2}} \tilde{\sigma}^{1}\left(\frac{\overline{A B}}{2}, x_{2}\right) \vec{n}_{1} d x_{2}+\int_{-\frac{\overline{A H}}{2}}^{0} \tilde{\sigma}^{2}\left(\frac{\overline{A B}}{2}, x_{2}\right) \vec{n}_{1} d x_{2}, \\
& \mathbf{S}_{1}^{2}\left(\gamma_{S_{i}}\right)=\int_{0}^{\frac{\overline{A B}}{2}} \tilde{\sigma}^{2}\left(x_{1},-\frac{\overline{A H}}{2}\right) \vec{n}_{2} d x_{1}+\int_{-\frac{\overline{A F}}{2}}^{0} \tilde{\sigma}^{3}\left(x_{1},-\frac{\overline{A H}}{2}\right) \vec{n}_{2} d x_{1} \\
& \mathbf{S}_{1}^{3}\left(\gamma_{S_{i}}\right)=\int_{0}^{\overline{A D}} \tilde{\sigma}^{4}\left(-\frac{\overline{A F}}{2}, x_{2}\right) \vec{n}_{3} d x_{2}+\int_{-\frac{\overline{A H}}{2}}^{0} \tilde{\sigma}^{3}\left(-\frac{\overline{A F}}{2}, x_{2}\right) \vec{n}_{3} d x_{2}, \\
& \mathbf{S}_{1}^{4}\left(\gamma_{S_{i}}\right)=\int_{0}^{\frac{\overline{A B}}{2}} \tilde{\sigma}^{1}\left(x_{1}, \frac{\overline{A D}}{2}\right) \vec{n}_{4} d x_{1}+\int_{-\frac{\overline{A F}}{2}}^{0} \tilde{\sigma}^{4}\left(x_{1}, \frac{\overline{A D}}{2}\right) \vec{n}_{4} d x_{1}
\end{aligned}
$$

The strain tensor in the Hooke's law is replaced by a local piecewise approximation, so that for each 1face $\gamma_{D_{i}}$ of $\gamma_{P}$, equation (3) becomes:

$$
\tilde{\sigma} \approx\left[\lambda\left(\operatorname{tr}\left(\frac{\tilde{d}+\tilde{d}^{T}}{2}\right)\right)-\kappa p\right] I+\mu\left(\tilde{d}+\tilde{d}^{T}\right)
$$

where the “ " sign stands for a local approximation of a given variable.

In order to express equation (12) at a local level, the strain tensor $\tilde{d}$ must be calculated for each location within $\gamma_{P}$. Since $\tilde{d}$ is derived from the configuration variable $\vec{\psi}$ (see equation (5)), the latter must be locally known. In the previous sections, the 0 -cochain $\boldsymbol{\Psi}_{0}$ was associated with the 0 -pixels of $K^{p}$; hence, $\vec{\psi}$ is derived using a bilinear interpolation of order 1 over $\gamma_{P}$. The bilinear interpolation of $\boldsymbol{\Psi}_{0}$ over $\gamma_{P}$ can be expressed as follows [5]: 


$$
\vec{\psi}\left(x_{1}, x_{2}\right)=\sum_{i=1}^{2}\left(\psi_{i, 00}+\left(\begin{array}{c}
\frac{\psi_{i, 00}-\psi_{i, 10}}{\overline{A B}} \\
\frac{\psi_{i, 00}-\psi_{i, 01}}{\overline{A D}} \\
\frac{\psi_{i, 00}+\psi_{i, 11}-\psi_{i, 01}-\psi_{i, 10}}{\overline{A B} \overline{A D}}
\end{array}\right)^{\mathrm{T}}\left(\begin{array}{c}
-x_{2} \\
-x_{1} \\
x_{1} x_{2}
\end{array}\right) \vec{e}_{i}\right.
$$

where the configuration variable at the $p q^{\text {th }} \quad 0$-pixel is given by $\vec{\psi}_{p q}=\left(\psi_{1, p q}, \psi_{2, p q}\right)^{T}$. Finally, the external forces cochain can be rewritten in terms of the configuration variable on the 0-pixels surrounding $\gamma_{F}$, as follows:

$$
\mathbf{F}_{2}\left(\gamma_{F}\right)=-\sum_{i=1}^{4} \int_{\gamma_{S_{i}}} \tilde{\sigma} \vec{n}_{i} d l
$$

Equation (14) can be considered as a linear algebraic system that can be rewritten as the product of a matrix encoding the basic physical laws and a vector containing the configuration variable values:

$$
\mathbf{F}_{2}\left(\gamma_{F}\right)=\left(\begin{array}{l}
F_{1}^{e x t} \\
F_{2}^{e x t}
\end{array}\right)=\left[\begin{array}{llll}
k_{1,1} & k_{1,2} & \cdots & k_{1,18} \\
k_{2,1} & k_{2,2} & \cdots & k_{2,18}
\end{array}\right]\left[\begin{array}{c}
\psi_{1,00}^{r} \\
\psi_{2,00}^{r} \\
\psi_{1,01}^{r} \\
\psi_{2,01}^{r} \\
\vdots \\
\psi_{1,-1-1}^{r} \\
\psi_{2,-1-1}^{r}
\end{array}\right]=[k][\psi](15)
$$

where $[\psi]$ is a vector whose elements are the configuration variable, on each 0-pixel of $K^{p}$ surrounding $\gamma_{F}$, in directions $x_{1}$ and $x_{2}$, and $[k]$ is a $2 \times 18$ matrix that encodes the basic laws in Figure 3 .

$$
\begin{gathered}
k_{1,1}=k_{2,2}=\left(-\frac{9}{2} \mu+\frac{3}{2} \lambda\right) \\
k_{1,3}=k_{1,9}=k_{2,4}=k_{2,10}=\left(\frac{1}{4} \mu-\frac{1}{4} \lambda\right) \\
k_{1,5}=k_{1,11}=k_{2,6}=k_{2,12}=\left(\frac{5}{4} \mu-\frac{3}{4} \lambda\right) \\
k_{1,7}=k_{1,13}=k_{1,15}=k_{1,17}=k_{2,8}=k_{2,14}=k_{2,16}=k_{2,18}=\left(\frac{3}{8} \mu+\frac{1}{8} \lambda\right) \\
k_{1,8}=k_{1,14}=k_{1,16}=k_{1,18}=k_{2,7}=k_{2,13}=k_{2,15}=k_{2,17}=\left(\frac{1}{4} \mu+\frac{1}{4} \lambda\right) \\
k_{1,2}=k_{1,4}=k_{1,6}=k_{1,10}=k_{1,12}=k_{2,1}=k_{2,3}=k_{2,5}=k_{2,9}=k_{2,11}=0
\end{gathered}
$$

In an image deformation problem, the external forces are calculated from the local image gray level and depend on the problem we are dealing with. Knowing the material characteristics $\lambda$, $\mu$, and $\kappa$, the solution of the image deformation problem consists of finding the elements of $[\psi]$ in equation (15). To accomplish this task, the system in equation (15) must also be solved for all 2-pixels from $K^{s}$ surrounding $\gamma_{F}$. Hence, the 
algebraic system valid for $\gamma_{F}$ is generalized for all 2-pixels of $K^{s}$, using a matrix assembling technique. Assuming that $K^{s}$ contains $n \times m$ 2-pixels, the generalized form can be written as follows:

$$
\left[F^{e x t}\right]=[K][\Psi]
$$

where $\left[F^{e x t}\right]$ and [ $\left.\Psi\right]$ are $2 n \times m$ vectors whose elements are respectively the values of the external force, on each 2-pixel of $K^{s}$, and the configuration variable, on each 0-pixel of $K^{p}$, in directions $x_{1}$ and $x_{2}$, and $[K]$ is a $(2 n \times m) \times(2 n \times m)$ matrix that encodes the basic physical laws. The system above can be solved either directly or using any iterative scheme.

In order to study image deformation, one must specify the physical nature of the image. The constitutive law in equation (5) is valid for both elastic materials and viscous fluids; hence, it is possible to express the deformation in both cases using the unified model introduced in the previous sections.

\section{A) The elastic material case}

The constitutive equation for a perfect elastic material is obtained by instantiating the parameters and variables in equation (5) as follows:

$$
\left\{\begin{array}{l}
\vec{\psi}=\vec{u} \\
\lambda=\lambda_{0} \\
\mu=\mu_{0} \\
\kappa=0
\end{array}\right.
$$

where $\vec{u}$ denotes the displacement field expressed and $\lambda_{0}$ and $\mu_{0}$ are the Lamé constants.

\section{B) Application to a viscous fluid}

Unlike an elastic material, a viscous fluid can be continuously deformed, since the internal forces developed are not directly linked to the displacements. Hence, such a model allows the image to undergo large-scale displacements. The constitutive equation for a viscous fluid is obtained by instantiating the parameters and variables in equation (5) as follows:

$$
\left\{\begin{array}{l}
\vec{\psi}=\vec{v} \\
\kappa=1
\end{array}\right.
$$

where $\vec{v}$ denotes the velocity field. The parameters $\lambda$ and $\mu$ in equation (5) are called the viscous fluid constants. Once the velocity at a given time $t$ is determined, the resulting displacement can be calculated using the discrete material equation given by:

$$
\vec{u}_{t+1}=\vec{u}_{t}+\Delta t\left(I-\left(\vec{\nabla} \vec{u}_{t}\right)^{T}\right) \vec{v}_{t}
$$

\section{$5 \quad$ Applications and Experimental Results}

Our deformation approach will be first tested in the context of optical flow estimation. In this case, the displacement is calculated from equation (15), using an external force that is derived from the optical flow constraints. The second application concerns neuroanatomy registration. In this case, the brain tissues are assumed to be viscous fluids that are deformed according to equation (15). 


\subsection{Optical flow estimation using elastic deformation}

A fundamental problem in the processing of image sequences is the computation of the 2D optical flow resulting from the projection of 3D object displacements onto the image plane. The estimated optical flow can be used to perform various tasks such as inferring the 3D motion and estimating the camera and scene parameters. The more widely cited works on optical flow estimation have been compiled in [6]. In what follows, the optical flow will be estimated using the CAT-based image deformation algorithm described in section 4. The objective is to derive an external force, which serves to estimate the optical flow through equation (15).

Let $I_{1}$ and $I_{2}$ be two consecutive frames from an image sequence. At a given location $\left(x_{1}, x_{2}\right)$, we define $\vec{d}=\left(d_{x_{1}}, d_{x_{2}}\right)$ as being the spatial shift that ensures the best matching between the image structures of $I_{1}$ and $I_{2}$. We consider that the best matching should minimize a distance measure (over $\vec{d}$ ) given by the sumof-squared difference (SSD). Consider a 2-pixel $\gamma_{F}$ from $K^{s}$ centered at location $\left(x_{1}, x_{2}\right)$. The SSD corresponding to a shift $\vec{d}$ is given as follows:

$$
T\left(x_{1}, x_{2}, d_{x_{1}}, d_{x_{2}}\right)=\sum_{(i, j) \in V(W)} W(i, j)\left[I_{1}\left(x_{1}+i, x_{2}+j\right)-I_{2}\left(x_{1}+i+d_{x_{1}}, x_{2}+j+d_{x_{2}}\right)\right]^{2}
$$

where $V(\cdot)$ is the support of a template $W$ centered on $\left(x_{1}, x_{2}\right)$ :

$$
\begin{cases}W(i, j)=1, & \text { if } \quad\left(x_{1}+i, x_{2}+j\right) \in \gamma_{F} \\ W(i, j)=0, & \text { elsewhere }\end{cases}
$$

The external force in equation (15), which will deform $I_{1}$ onto $I_{2}$, is defined as being the gradient of the distance measure:

$$
\vec{F}_{0}^{e x t}=-\left(\frac{\partial T}{\partial d_{x_{1}}}, \frac{\partial T}{\partial d_{x_{2}}}\right)^{T}
$$

However, this is not sufficient to yield reliable estimation of optical flow, since the edge information is not handled, which may produce a misalignment between contours from $I_{1}$ and $I_{2}$. We therefore define additional distances, measuring the SSD between the first order derivatives of $I_{1}$ and $I_{2}$ in both $x_{1}$ and $x_{2}$ directions:

$$
\begin{aligned}
& T_{x_{1}}\left(x_{1}, x_{2}, d_{x_{1}}, d_{x_{2}}\right)=\sum_{i=-l}^{i=l} \sum_{j=-l}^{j=l} W(i, j)\left[\frac{\partial I_{1}}{\partial x_{1}}\left(x_{1}+i, x_{2}+j\right)-\frac{\partial I_{2}}{\partial x_{1}}\left(x_{1}+i+d_{x_{1}}, x_{2}+j+d_{x_{2}}\right)\right]^{2} \\
& T_{x_{2}}\left(x_{1}, x_{2}, d_{x_{1}}, d_{x_{2}}\right)=\sum_{i=-l}^{i=l} \sum_{j=-l}^{j=l} W(i, j)\left[\frac{\partial I_{1}}{\partial x_{2}}\left(x_{1}+i, x_{2}+j\right)-\frac{\partial I_{2}}{\partial x_{2}}\left(x_{1}+i+d_{x_{1}}, x_{2}+j+d_{x_{2}}\right)\right]^{2}
\end{aligned}
$$

The external force attached with the distances in equations (21) and (22) is thus defined as follows: 


$$
\vec{F}_{1}^{e x t}=-\left(\frac{\partial T_{x_{1}}}{\partial d_{x_{1}}}, \frac{\partial T_{X_{2}}}{\partial d_{x_{2}}}\right)^{T}
$$

Let $\quad \overrightarrow{\widetilde{d}}_{0}\left(x_{1}, x_{2}\right)=\left(\tilde{d}_{x_{1}}^{0}, \tilde{d}_{x_{2}}^{0}\right)^{\mathrm{T}}$ and $\overrightarrow{\widetilde{d}}_{1}\left(x_{1}, x_{2}\right)=\left(\tilde{d}_{x_{1}}^{1}, \tilde{d}_{x_{2}}^{1}\right)^{\mathrm{T}}$ be the estimated shift maps using $\vec{F}_{0}^{\text {ext }}$ and $\vec{F}_{1}^{\text {ext }}$ respectively. The next step consists of locally merging these maps to generate a solution $\overrightarrow{\widetilde{d}}$ that ensures an optimal fit between $I_{1}$ and $I_{2}$ according to equation (19):

$$
\overrightarrow{\widetilde{d}}\left(x_{1}, x_{2}\right)=\underset{p}{\arg \min }\left(\sum_{i=-l}^{i=l} \sum_{j=-l}^{j=l}\left[I_{1}\left(x_{1}+i, x_{2}+j\right)-I_{2}\left(x_{1}+i+d_{x_{1}}^{p}, x_{2}+j+d_{x_{2}}^{p}\right)\right]^{2}\right)
$$

In order to allow tracking of large displacements between frames, a multi-resolution scheme is used. Unlike [7][8] where a coarse-to-fine approach is favored, we propose to compute the shift map at each resolution independently and to merge the maps thus obtained, using equation (24). In this way, errors occurring at a coarser resolution will not be propagated to finer levels. We examined the performance of our algorithm on image pairs (see figure 5) with known motion fields (see left columns of figures 6,7 and 8). The translating tree sequence is generated by moving the camera along its $X$-axis. The diverging tree sequence is generated by moving the camera along its line of sight with the focus of expansion located at the center of the image. The Yosemite sequence contains translating and diverging motions.

In all the experiments, the Lamé constants were tuned by trial-and-error process. The values which experimentally give the best results are $\lambda_{0}=5$ and $\mu_{0}=20$. The matching was carried out for three levels of resolution corresponding to side lengths of the $K^{p}$ 2-pixels equal to 3, 5 and 7. The estimated flow maps are given in the right columns of figures 6,7 and 8 . In order to quantify the accuracy of the maps obtained, we computed the angular error and its standard deviation. This measure provides the angular deviation between the computed and the correct maps. For each sequence, we compared the accuracy of the computed flow map with the best accuracy obtained on the same sequence using the algorithms reported in [6]. (These algorithms are described in [9][10][11][12].) The multiple comparisons were motivated by the fact that no single algorithm performed best on all sequences. Visual inspection of the obtained results together with the accuracy measures provided in table 1 show that our method gives a precise estimation and that the CATbased elastic constraint ensures smooth motion fields. Since the diverging tree sequence has a wide range of displacements, the estimation of flow map for this sequence could be easily improved by using an external force that handles subpixel displacements.
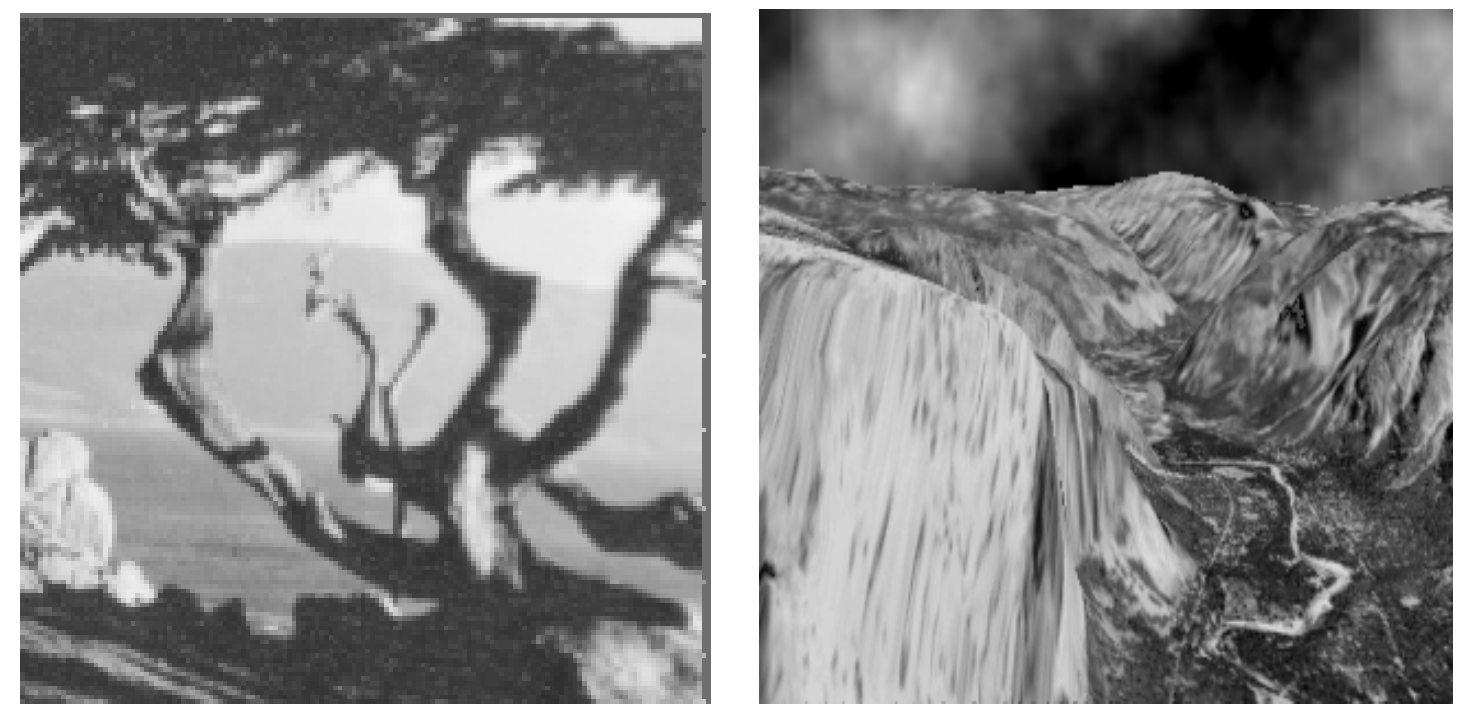

Fig. 5: left: Tree sequence; right: Yosemite sequence 

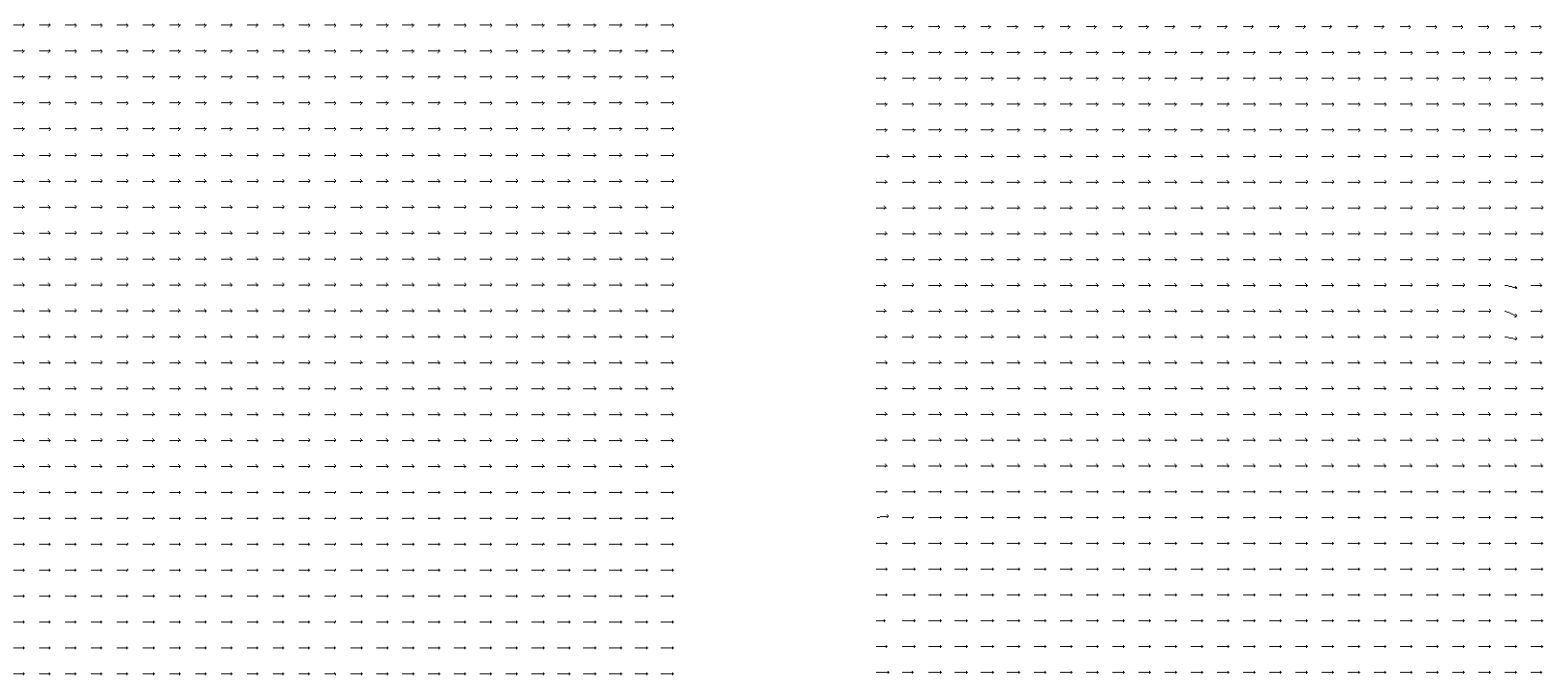

Fig. 6: Translating tree, left: ground truth; right: estimated flow map

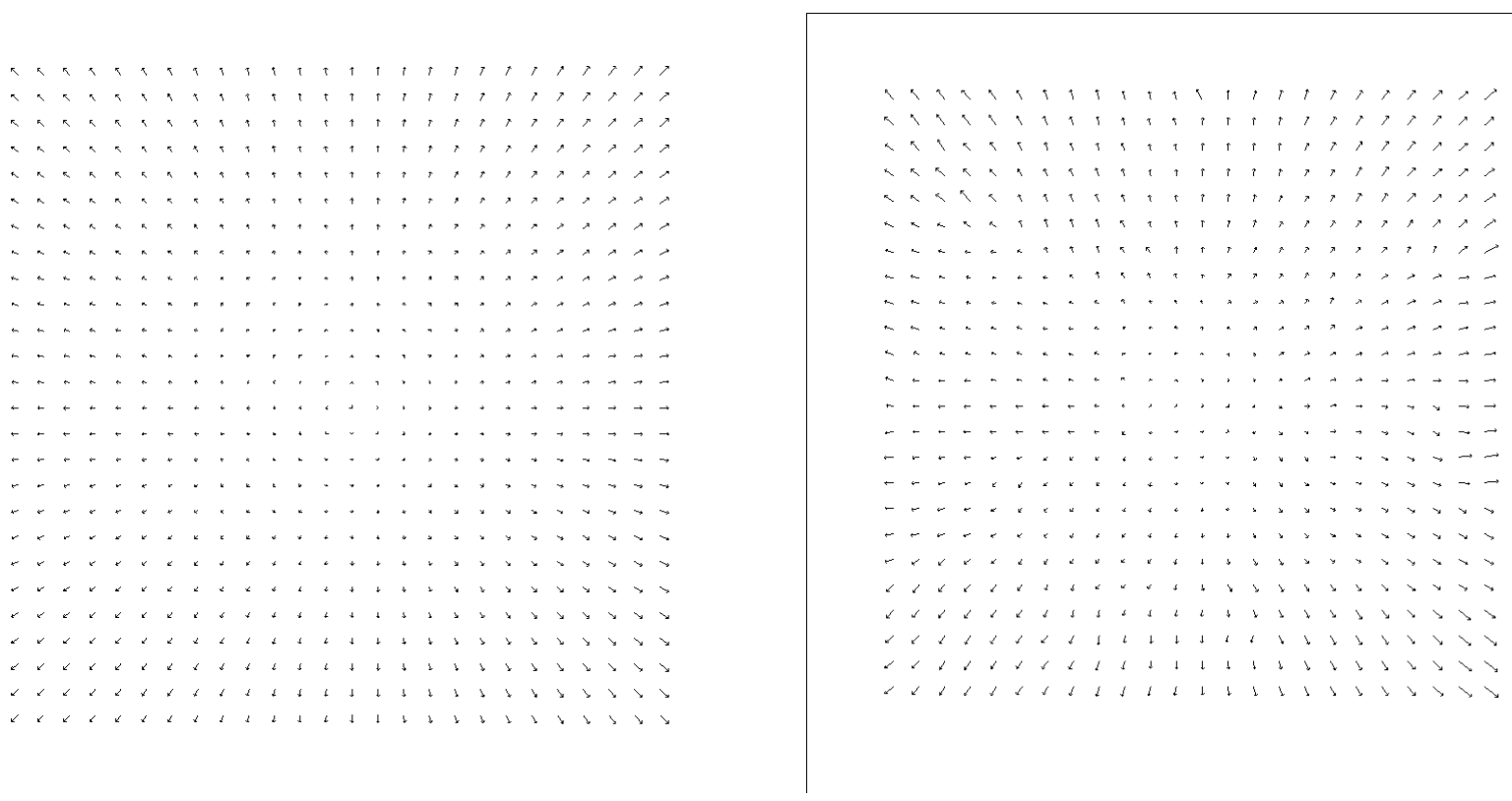

Fig. 7: Diverging tree, left: ground truth; right: the estimated flow map 

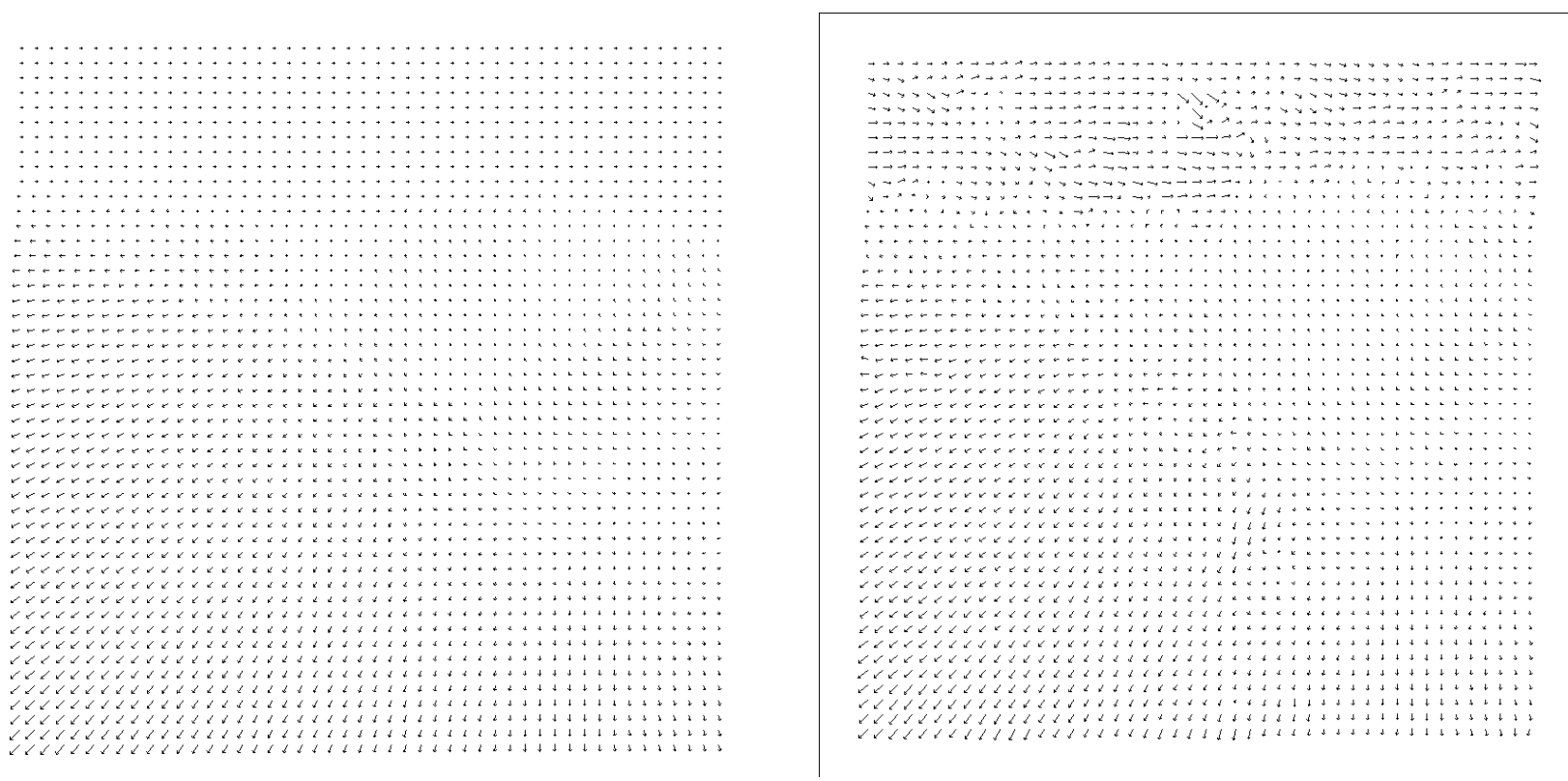

Fig. 8: Yosemite, left: ground truth; right: the estimated flow map

Tab. 1: Mean and standard deviation of the angular error

\begin{tabular}{ccccccc} 
& \multicolumn{2}{c}{ Translating tree } & \multicolumn{2}{c}{ Diverging tree } & \multicolumn{2}{c}{ Yosemite sequence } \\
\hline & Mean error & $\begin{array}{c}\text { Standard } \\
\text { deviation }\end{array}$ & Mean error & $\begin{array}{c}\text { Standard } \\
\text { deviation }\end{array}$ & Mean error & $\begin{array}{c}\text { Standard } \\
\text { deviation }\end{array}$ \\
\hline Our method & $1.14^{\circ}$ & $1.09^{\circ}$ & $2.81^{\circ}$ & $2.72^{\circ}$ & $7.80^{\circ}$ & $7.91^{\circ}$ \\
Best from [6] & $1.25^{\circ}$ & $3.29^{\circ}$ & $2.55^{\circ}$ & $3.67^{\circ}$ & $10.44^{\circ}$ & $15^{\circ}$ \\
\hline
\end{tabular}

\subsection{D brain registration using fluid deformation}

In neuroanatomy registration, the main objective is to match an image $I_{1}$ called the template to another image $I_{2}$ called the study. The study is usually a set of images representing a healthy brain. The registration of a patient's brain onto an atlas consists of finding the set of geometrical transformations that must be applied to the atlas to fit the patient's brain images [13]. Once the transformations are known, all of the information contained in the atlas, such as structure names, sizes, locations, and atlas segmentation, is mapped onto the patient's brain images. One of the most cited work in the literature is the viscous fluid model proposed by Christensen et al. [14]. In their work, Christensen et al. proposed to solve the following PDE:

$$
\mu \nabla^{2} \vec{v}+(\lambda+\mu) \vec{\nabla}(\vec{\nabla} \cdot \vec{v})=\vec{F}^{e x t}
$$

An iterative scheme is then derived to solve equation (25) using the successive over-relaxation (SOR) with checkerboard update method [14]. This approach will be compared to the CAT-based image deformation as described in section 4 . Hence, the brain images will be considered as viscous fluids. The external force in equation (15), which deforms the template onto the study, is derived from the derivative of a Gaussian sensor model and defined as follows:

$$
\vec{F}_{n}^{e x t}=-\left(I_{1}\left(x_{1}, x_{2}\right)-I_{2}\left(x_{1}, x_{2}\right)\right) \vec{\nabla} I_{1}
$$


where $\vec{\nabla} I$ is the gradient of $I$. The external force in the equation above attempt to make edges in the template and the study fit. Note that the use of such a force requires the template to be initially aligned with the study so that they overlap. Experiments on brain images will be presented below. The resulting registration and time processing are compared with those obtained using the SOR algorithm. In all experiments, we assume that the template and the study contain the same structures. $\lambda$ and $\mu$ are set at 1 and 5, respectively. The experiments were performed on a sample of CT scan images measuring $256 \times 256$ pixels. The left column in figure 9 shows the templates and the right column the studies. Pairs (A) and (C), are not equivalent since they do not contain the same configuration of grey/white matter. The deformed templates obtained using the CAT-based algorithm are shown in the middle column. Notice that the algorithm accommodates local shape variations and large-scale deformations with a good level of detail. However, there are some situations where the algorithm cannot recover the study shape, such as the grey matter in pair (A). This is mainly due to violation of the initial assumptions concerning similarity of the white/grey matter configuration. A 3D registration can overcome this problem, since the information from other slices will make the template and the study match. The second column in table 2 indicates the mean and standard deviation of the grey-level difference between the deformed templates and the studies for the CAT-based algorithm. The third column indicates the same measures when the SOR algorithm is employed. The obtained measures indicate that the CAT-based algorithm yields the best performance in terms of accuracy. Moreover, it is important to mention that the CAT-based algorithm takes only 10 iterations to perform the deformation, whereas the SOR requires 250 iterations to solve equation (25) and 100 iterations to accomplish the registration.

Tab. 2: Mean and standard deviation of the gray-level difference between the deformed template and the study

\begin{tabular}{ccc}
\hline Experiment & Our method & SOR method \\
\hline A & $\mu=6.0 \sigma=12.6$ & $\mu=10.9 \sigma=19.2$ \\
B & $\mu=7.9 \sigma=13.7$ & $\mu=9.7 \sigma=18.1$ \\
C & $\mu=6.8 \sigma=13.8$ & $\mu=9.3 \sigma=17.2$ \\
D & $\mu=6.2 \sigma=13.0$ & $\mu=9.1 \sigma=17.1$ \\
E & $\mu=6.2 \sigma=13.2$ & $\mu=9.1 \sigma=16.6$ \\
F & $\mu=5.9 \sigma=12.5$ & $\mu=13.2 \sigma=20.1$ \\
\hline
\end{tabular}

\section{Conclusion}

In this paper, we have presented a new approach for image deformation utilizing elasticity and viscous fluid models. In the proposed approach, the image model is based on algebraic topology. This allows us to decompose the image deformation problem into one of basic physical laws. Cochains encode these laws over complexes and are linked together using coboundaries and codual operators. The major advantage of such an approach lies in the possibility of solving the deformation problem directly from the exact global forms rather than from discrete differential forms. Consequently, errors resulting from the approximation of continuous fields (i.e., displacement and velocity) and derivative operators by discrete forms can be reduced. Furthermore, the idea of expressing the physics-based deformation in a modular way is very interesting since it can be applied to solve other computer vision problems based on a physical interpretation. CAT-based deformation under the elasticity assumption was tested successfully for the optical flow estimation. The results show that our algorithm yields increased stability and is more efficient than several existing algorithms. The same statement is valid for neuroanatomy registration, where a CAT-based viscous fluid model was used. 
(A)

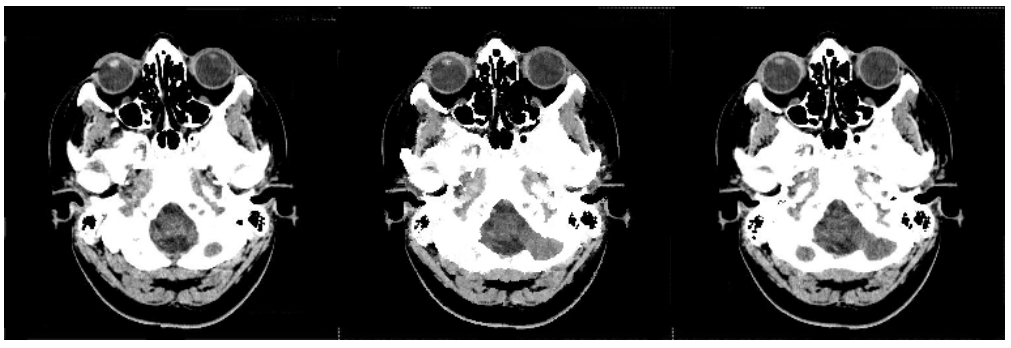

(B)

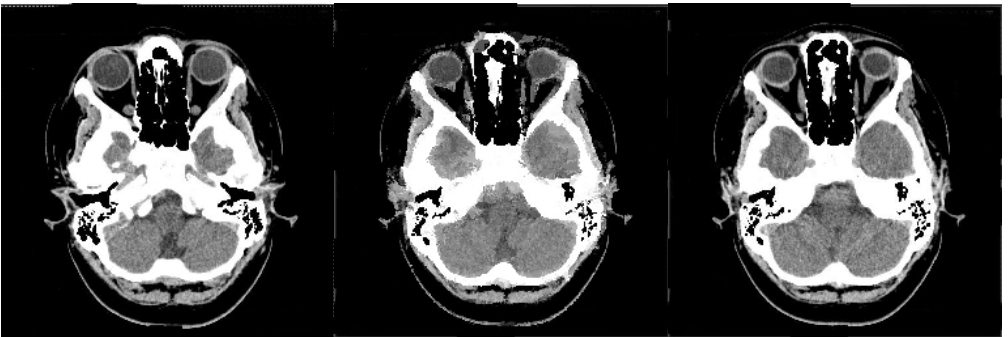

(C)

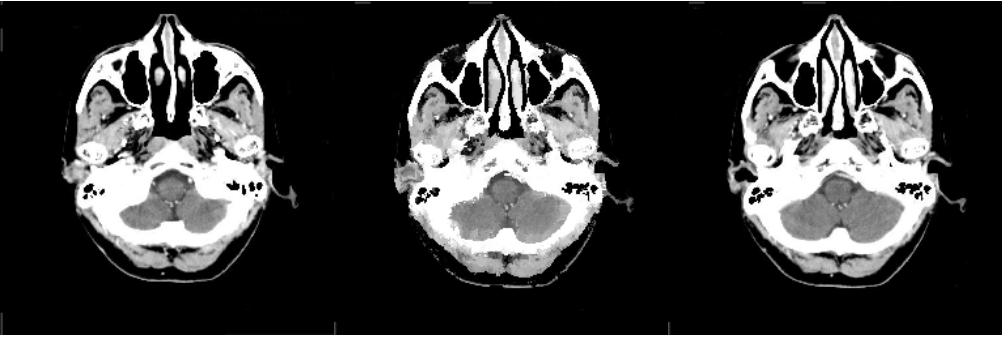

(D)

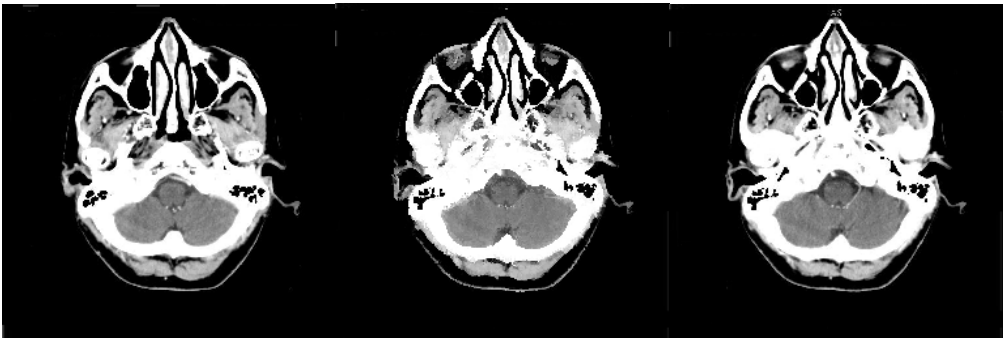

(E)

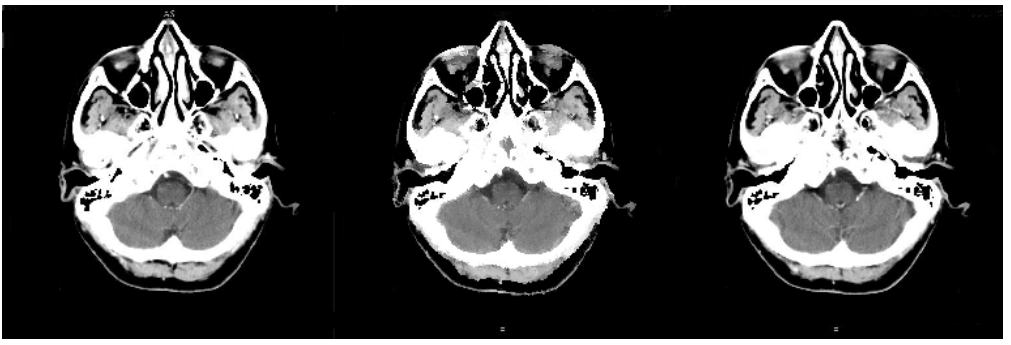

(F)
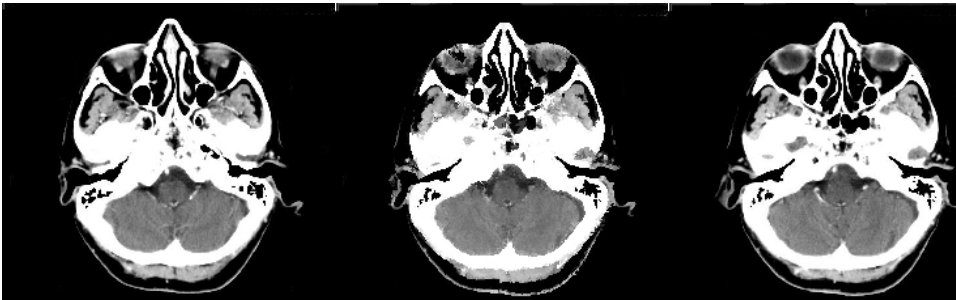

Fig. 9: Left: template; middle: after viscous fluid deformation; right: study 


\section{References}

[1] M. Kass, A. Witkin, and D. Terzopoulos, "Snakes: Active contour models," International Journal of Computer Vision, vol. 1, no. 4, pp. 321-331, 1988

[2] F. Cosmi, "Material response with the cell method," Proceedings of the $10^{\text {th }}$ International Conference on Fracture, Hawaii, 2001.

[3] D. Ziou and M. Allili, "Generating cubical complexes from image data and computation of the Euler number,” Pattern Recognition, vol. 35, no. 12, pp. 2833-2939, December 2002.

[4] A. P. Boresi, Elasticity in Engineering Mechanics, Prentice-Hall, 1965.

[5] M.-F. Auclair-Fortier, P. Poulin, D. Ziou and M. Allili, "A Computational Algebraic Topology Approach for Diffusion Process," 3rd Workshop on Physics in Signal and Image Processing, pp 77-80, Grenoble, France, January 2003.

[6] J. L. Barron, D. J. Fleet, S. S. Beauchemin, and T. A. Burkitt, "Performance of optical flow techniques," International Journal of Computer Vision, vol. 12, no. 1, pp. 43-77, 1994.

[7] D. J. Heeger, “Optical flow using spatiotemporal filters,” International Journal of Computer Vision, vol. 1, no. 4, pp. 279-302, 1988.

[8] A. Singh, "An estimation-theoretic framework for image-flow computation," Proceedings of IEEE ICCV, pp. 168-177, Osaka, 1990.

[9] B. K. P. Horn, and B. G. Schunck, "Determining optical flow,” Artificial Intelligence, vol. 17, pp 185204, 1981.

[10] H. H. Nagel, "On a constraint equation for the estimation of displacement rates in image sequences," IEEE Transactions on Pattern Analysis and Machine Intelligence, vol. 11, no.1, pp. 13-30, 1989.

[11] P. Anandan, "A computational framework and an algorithm for the measurement of visual motion," International Journal of Computer Vision, vol. 2, no. 3, pp. 283-310, 1989.

[12] P. T. Fox, J. S. Perlmutter, and M. E. Raichle, “A stereotactic method of anatomical localization for position emission tomography," Journal of Computational Assisted Tomography, vol. 9, no. 1, pp. 141153, 1985.

[13] H. Lesten, S. R. Aridge, “A survey of hierarchical non-linear medical image registration,” Pattern Recognition, vol. 32, no. 1, pp. 129-149, 1999.

[14] G. E. Christensen, S. Joshi, and M. I. Miller, "Volumetric transformation of brain anatomy," IEEE Transactions on Medical Imaging, vol. 16, no. 6, pp. 864-877, 1997 